\title{
Face Recognition Based Attendance System with Auto Alert to Guardian Using Call and SMS
}

\author{
Diksha Ghare, Prajakta Katakdhond, Shraddha Ujgare, Komal Suskar, Prof. Amruta Surana \\ Department of Computer Engineering, PCET's NMIET Talegaon-Dabhade, Maharashtra, India
}

\section{ABSTRACT}

Now a days the smart attendance management system using face detection techniques. Daily attendance marking is a common and important activity in schools and colleges for checking the performance of students. Manual Attendance maintaining is difficult process, especially for large group of students. Some automated systems developed to overcome these difficulties, have drawbacks like cost, fake attendance, accuracy, intrusiveness. To overcome these drawbacks, there is need of smart and automated attendance system. We are implementing attendance system using face recognition. Since face is unique identity of person, the issue of fake attendance and proxies can be solved.

Key Words: Face Authentication, Viola and Jones Algorithm, SMS, Auto Call

\section{INTRODUCTION}

Today's dynamic business environment requires realtime time-attendance and access control solution with anytime, anywhere access. The existing biometric credentials used for authentication faces some challenges in identification due to various biometric changes and extreme weather condition effect on biometrics. Matrix presents COSEC Face Recognition - A Powerful Biometric User Identification based on Innovative, Deep Learning Technology. It accepts the user's mobile as a credential and evolves as per regular user interaction in different conditions. The technology checks liven of a face with $99.53 \%$ accuracy. It is a facial recognition attendance system that ensures contactless authentication and identifies user's face in $<1 \mathrm{Sec}$. Our hi-tech world has brought to us so many machineries which made our lives quite easy.

\section{Motivation}

The main Motivation of this Attendance System should be Automated calls and SMS to Guardian. Attendance based on Face Detection. No proxy attendance can be get marked as we are going to record attendance of student's by recognizing their faces And after recognizing the student face his/her attendance will get marked. As face is the unique identity of person.

\section{Literature Survey}

Paper1. Algorithm for Efficient Attendance Management: Face Recognition based approach

Author Name: Naveed Khan Balcoh, M. HaroonYousaf, Waqar Ahma nd M. IramBaig

Description: All These methods also waste time because students have to make a queue to touch their thumb on the scanning device. This work describes the efficient algorithm that automatically marks the Attendance without human intervention. This attendance is recorded by using a camera attached in front of classroom that is continuously capturing images of students, detect the faces in images and compare the detected faces with the database and mark the attendance. The paper review the related work in the field of attendance system then describes the system architecture, software algorithm and results.

Paper2. Study of implementing Automated Attendance System using Implementing Automated Attendance System Using face recognition Technique Author Name: Nirmalaya Kar, Mrinal Kanti Debbarma, AshimSaha, and Dwijen Rudra Pal,

Description: Authentication is a significant issue in system control in computer based communication. Human face recognition is an important branch of biometric verification and has been widely used in many applications, such as video monitor system, 
human-computer interaction, and door control system and network security. This paper describes a method for Student's Attendance System which will integrate with the face recognition technology using Personal Component Analysis (PCA) algorithm. The system will record the attendance of the students in class room environment automatically and it will provide the facilities to the faculty to access the information of the students easily by maintaining a log for clock-in and clock-out time.

Paper3. Face Recognition using Principal Component Analysis in MATLAB

Author Name: P Singh, A Sharma

Description: The papers present an semi-automated program for human face recognition. A self prepared database of different faces is used. Task of removing background from the image is a challenge but on the other hand by implementing Viola-Jones face detection algorithm and by Principal Component analysis it is possible. An application of system can be real time implementation of face recognition system. A robust and reliable form of recognition can be done by using Principal Component analysis.

Paper4. Face recognition using eigen faces

Author Name: M. Turk and A. Pentland

Description: We present an approach to the detection and identification of human faces and describe a working, near-real-time face recognition system which tracks a subject's head and then recognizes the person by comparing characteristics of the face to those of known individuals. Our approach treats face recognition as a two-dimensional recognition problem, taking advantage of the fact that faces are are normally upright and thus may be described by a small set of 2-D characteristic views. Face images are projected onto a feature space ("face space") that best encodes the variation among known face images. The face space is defined by the "eigenfaces", which are the eigenvectors of the set of faces; they do not necessarily correspond to isolated features such as eyes, ears, and noses. The framework provides the ability to learn to recognize new faces in an unsupervised manner.

\section{Existing System}

In the existing system the system only detect Images and save in the database for attendance system. In the existing system face recognition the recognition of the face is direct without removing any noise and absent record were not send to guardian.
Attendance management is important to every single organization; it can decide whether or not an organization such as educational institutions, public or private sectors will be successful in the future. Organizations will have to keep a track of people within the organization such as employees and students to maximize their performance. Managing student attendance during lecture periods has become a difficult challenge. The ability to compute the attendance percentage becomes a major task as manual computation produces errors, and wastes a lot of time. Authentication is a significant issue in system control in computer based communication. Human face recognition is an important branch of biometric verification and has been widely used in many applications, such as video monitor system, human-computer interaction, and door control system and network security. This paper describes a method for Students Attendance System which will integrate with the face recognition technology using Personal Component Analysis algorithm. The system will record the attendance of the students in class room environment automatically and it will provide the facilities to the faculty to access the information of the students easily by maintaining a log for clock-in and clock-out time.

\section{Architecture of proposed system}

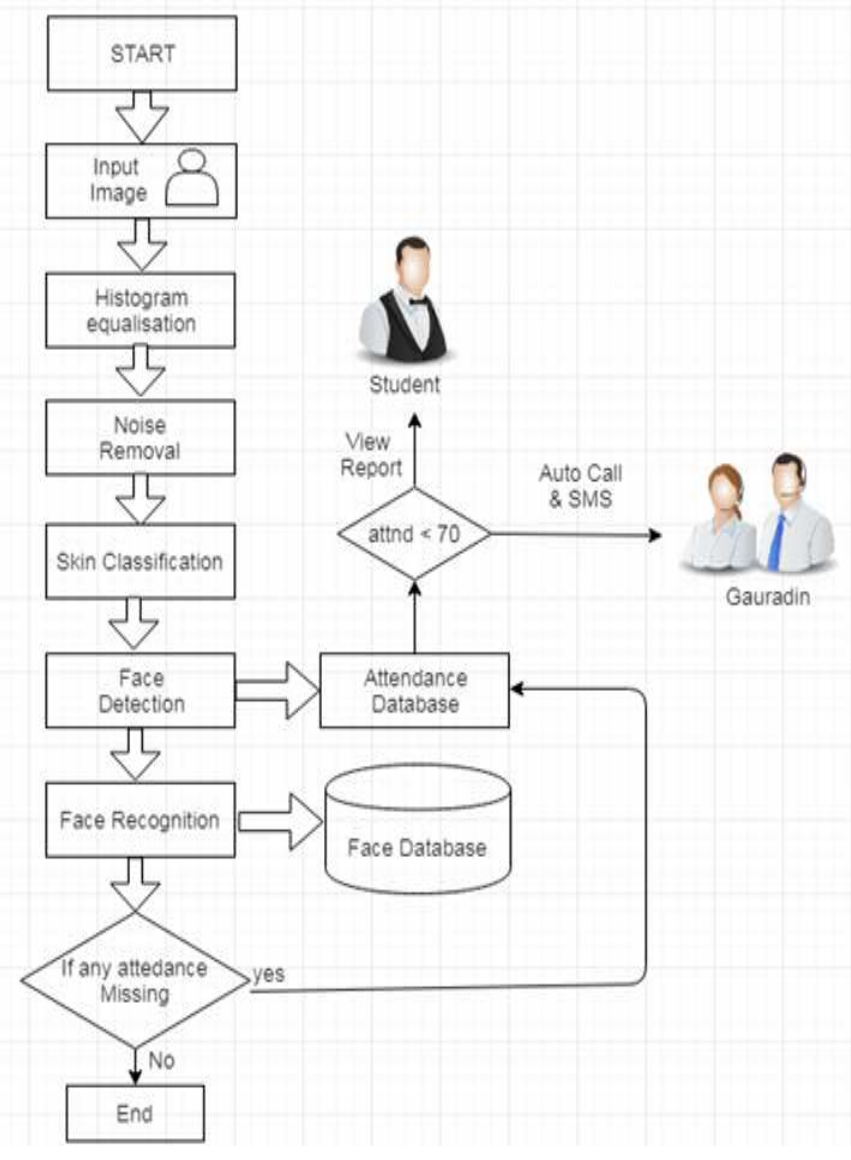




\section{Propose System}

The experimental setup Patterns of face pictures of an single student is stored in the databank at time of registration. so the faces are then recognised from that of the image then the procedure matches with the database. The attendance marked on server if any of the face identified from the database and it can be accessed by everyone for numerous reasons. This also habits the procedure that can be used for attendance. The system is also attached with the time table unit that robotically gets class at what time and what subject. The system robotically becomes presence deprived of opinions the learners and the teachers. So teachers after coming to the class when taking attendance they just press the attendance button to start the attendance procedure. This method is highly protected method here no one can give the attendance of the other and also saves a lot of time.

\section{Algorithm:}

\section{Viola-Jones Algorithm}

The Viola-Jones algorithm is a widely used mechanism for object detection. The main property of this algorithm is that training is slow, but detection is fast. This algorithm uses Haar basis feature filters, so it does not use multiplications.

The efficiency of the Viola-Jones algorithm can be significantly increased by first generating the integral image.

$$
I I(y, x)=\sum_{p=0}^{y} \ldots \sum_{q=0}^{x} Y(p, q)
$$

The integral image allows integrals for the Haar extractors to be calculated by adding only four numbers. For example, the image integral of area $\mathrm{ABCD}$ is calculated as $\mathrm{II}(\mathrm{yA}, \mathrm{xA})-\mathrm{II}(\mathrm{yB}, \mathrm{xB})-$ $\mathrm{II}(\mathrm{yC}, \mathrm{xC})+\mathrm{II}(\mathrm{yD}, \mathrm{xD})$.

\section{Conclusion}

Capturing the images from camera or cc camera and applying techniques face detection and recognition can decrease the manual work from human and increase the security safety, taking the decision from this recognition result. Based on this face detection and recognition can used in implement so many application like automatic attendances system based on face recognition, worker attendances, security, safety like finding thief in image that help to catching thief. In this system we have implemented an attendance system for a lecture, section or laboratory by which lecturer or teaching assistant an record students attendance. It saves time and effort, especially if it is a lecture with huge number of students. The complete system is implemented in JAVA. This attendance system shows the use of facial recognition techniques for the purpose of student attendance and for the further process this record of student can be used in Attendance related issues.

\section{Reference}

1. Naveed Khan Balcoh, M. HaroonYousaf, Waqar Ahma nd M. IramBaig, Algorithm for efficient Attendance Management: Face Recognition Based approach, International Journal of Computer Science Issue, Vol.9, Issue 4, No 1, July 2012.

2. NirmalayaKar, MrinalKantiDebbarma, AshimSaha, and DwijenRudraPal, Study of implementing Automated Attendance System using Implementing Automated Attendance System Using face recognition Technique, International Journal of Computer and Communication Engineering, Vol1, No 2,July 2012

3. O. Shoewn Development of Attendance Management System using Biometrics. The Pacific Journal of Science and Technology Volume 13, No 1, May 2012

4. M. Turk and A. Pentland (1991) "Face recognition using eigen faces". Proc. IEEE conference on computer vision and Pattern Recognition

5. W. Zhao, R. Chellapa, P. J. Phillips and A. Rosenfld, 'Face Recognition: A Literature Survey, vol. 35, No 4, Dec 2003, pp.399-458

6. R. L. Hsu, Mottalec M.A and A. K. Jain, "Face Detection in colour images", Proceedings International Conference on Image Processing, Oct 2001,pp. 1046-1049

7. ToufiqP. Ahmed Egammal and Anurag mittal (2006),"A Framework for feature selection for Background Subtraction", in Proceedings of IEEE computer Society Conference on Computer Vision and Pattern Recognition..

8. M. H. Yang, N. Ahuja and D. Kriegmao, "Face recognition using kernel eigenfaces", IEEE International Conference on Image Processing, vol.1, pp. 10-13, Sept. 2000 